\title{
ON SNAKE CONES, ALTERNATING CONES AND RELATED CONSTRUCTIONS
}

\author{
Katsuya EdA, Umed H. Karimov, Dušan Repovš and Andreas \\ ZASTROW
}

Waseda University, Japan, Academy of Sciences of Tajikistan, Tajikistan, University of Ljubljana, Slovenia and Gdansk University, Poland

\begin{abstract}
We show that the Snake on a square $S C\left(S^{1}\right)$ is homotopy equivalent to the space $A C\left(S^{1}\right)$ which was investigated in the previous work by Eda, Karimov and Repovš. We also introduce related constructions $C S C(-)$ and $C A C(-)$ and investigate homotopical differences between these four constructions. Finally, we explicitly describe the second homology group of the Hawaiian tori wedge.
\end{abstract}

\section{INTRODUCTION}

The functor $S C(-,-)$, mapping from the category of all spaces with base points and continuous mappings to the subcategory of simply connected spaces was constructed in [3]. We named $S C(X, x)$ the Snake cone over a pointed space $(X, x)$. In the case when the space $X$ is a circle $S^{1}$ with a base point $x$, the resulting space $S C\left(S^{1}, x\right)$, called the Snake on a square, is a cell-like simply connected 2-dimensional Peano continuum ([3]). It was shown in [4] that the space $S C\left(S^{1}, x\right)$ is not only noncontractible but is also nonaspherical (because the second homotopy group of this space is nontrivial, see also $[5,6])$. We investigated another functor $A C(-,-)$ in [7], which shares many properties with $S C(-,-)$, and we proved that $A C(\mathbb{H}, o)$ is not homotopy equivalent to $S C(\mathbb{H}, o)$ for the Hawaiian earring $\mathbb{H}$ with the distinguished point $o$. We named $A C(X, x)$ the Alternating cone over a pointed space $(X, x)$. In the present paper we shall introduce some variants of these constructions, i.e., the Collapsed snake cone $C S C(X, x)$ and the

2010 Mathematics Subject Classification. 54F15, 55N10, 54G20, 55Q52.

Key words and phrases. Noncontractible compactum, weak homotopy equivalence, trivial shape, Peano continuum, topologist sine curve, snake on a square, collapsed snake cone, collapsed alternating cone, asphericity, Hawaiian earring, Hawaiian tori. 
Collapsed alternating cone $C A C(X, x)$, and we shall investigate homotopy equivalences among these four functors.

Our main results are the following:

THEOREM 1.1. If a space $X$ is semi-locally strongly contractible at $x_{0} \in$ $X$, then $S C\left(X, x_{0}\right)$ and $C S C\left(X, x_{0}\right)$ (resp. $A C\left(X, x_{0}\right)$ and $C A C\left(X, x_{0}\right)$ ) are also homotopy equivalent.

TheOREM 1.2. The Snake on a square $S C\left(S^{1}, x_{0}\right)$ and the Alternating cone $A C\left(S^{1}, x_{0}\right)$ are homotopy equivalent for every $x_{0} \in S^{1}$.

TheOREM 1.3. For the Hawaiian earring $\mathbb{H}$ with the base point o the following properties hold:

(1) $S C(\mathbb{H}, o)$ and $A C(\mathbb{H}, o)$ (resp. $S C(\mathbb{H}, o)$ and $C S C(\mathbb{H}, o)$ ) are not homotopy equivalent;

(2) $A C(\mathbb{H}, o)$ and $C A C(\mathbb{H}, o)$ are not homotopy equivalent; but

(3) $C S C(\mathbb{H}, o)$ and $C A C(\mathbb{H}, o)$ are homotopy equivalent.

TheOREM 1.4. For the 2-dimensional torus $T$ with the base point $z_{0} \in$ $T$, the spaces $S C\left(T, z_{0}\right)$ and $A C\left(T, z_{0}\right)$ are not homotopy equivalent and consequently, also the spaces $C S C\left(T, z_{0}\right)$ and $C A C\left(T, z_{0}\right)$ are not homotopy equivalent.

Consequently, we get the following:

COROLlary 1.5. For each pair of functors $S C(-,-), A C(-,-)$, $C S C(-,-)$ and $C A C(-,-)$, there exists a space such that the resulting functorial spaces are not homotopy equivalent.

We shall define the Hawaiian tori wedge similarly to the Hawaiian earring by replacing the circle by the torus. A precise definition and supporting notions will be given in the forthcoming sections.

TheOREM 1.6. Let $\mathbb{H}_{T}$ be the Hawaiian tori wedge. Then the following properties hold:

(1) $\pi_{1}\left(\mathbb{H}_{T}\right)$ is isomorphic to the free $\sigma$-product of countable copies of the free abelian group of rank two;

(2) $\pi_{2}\left(\mathbb{H}_{T}\right)$ is trivial; and

(3) $H_{2}\left(\mathbb{H}_{T}\right)$ is isomorphic to the free abelian group on countably many generators. The generators are associated with the fundamental cycles of the tori.

This contrasts with the known results concerning the 2-dimensional Hawaiian earring $\mathbb{H}_{2}$. Namely,

(1) $\pi_{1}\left(\mathbb{H}_{2}\right)$ is trivial ([2, Theorem A.1]); and

(2) $\pi_{2}\left(\mathbb{H}_{2}\right) \cong H_{2}\left(\mathbb{H}_{2}\right)$ is isomorphic to the direct product of countably many copies of $\mathbb{Z}([8$, Corollary 1.2$])$. 
Throughout this paper $X$ stands for a path-connected compact Hausdorff space. Standard notions are undefined and we refer the reader to [11].

\section{The construction of the Snake cone $S C(X, x)$, the Alternating CONe $A C(X, x)$ And their Variations}

In this paper we shall apply our constructions only to compact spaces, and so the definitions of topologies, which we shall use, may look to be different from the original ones in [3], but they are in fact the same. The construction of the Snake cone is based on the piecewise-linear Topologist sine curve $\mathcal{T}$ which is homeomorphic to the usual Topologist sine curve. To describe this space and for the next discussion we need to fix some terminology. For any two points $A$ and $B$ in the plane $\mathbb{R}^{2}$, we denote by $[A, B]$ the linear segment connecting these points. The unit segment of the real line is denoted by $\mathbb{I}$. The point of the coordinate plane $\mathbb{R}^{2}$ with coordinates $a$ and $b$ is denoted as $(a ; b)$, particularly when we describe the points in $S C(X, x)$ and $A C(X, x)$. Let $A=(0 ; 0), B=(0 ; 1), A_{n}=(1 / n ; 0), B_{n}=(1 / n ; 1)$ be points and let $L=[A, B], L_{2 n-1}=\left[A_{n}, B_{n}\right], L_{2 n}=\left[B_{n}, A_{n+1}\right]$ be the segments in the plane $\mathbb{R}^{2}$ for $n \in \mathbb{N}=\{1,2,3, \ldots\}$. We also let $C_{2 n-1}=(1 / n ; 1 / 2)$ and $C_{2 n}=(1 /(n+1)+1 / 2 n(n+1) ; 1 / 2)$ for $n \in \mathbb{N}$ and $C=(0 ; 1 / 2)$, see Figure 1 .

The piecewise linear Topologist sine curve $\mathcal{T}$ is the subspace of $\mathbb{R}^{2}$ defined as the union of $L_{n}$ and $L$.

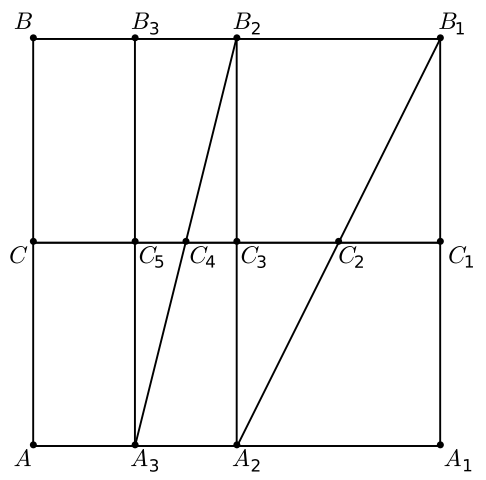

FiguRE 1. Topologist sine curve

The Snake cone $S C(X, x)$ over a compact pointed space $(X, x)$ is the quotient space of the topological sum $(X \times \mathcal{T}) \bigsqcup \mathbb{I}^{2}$ via the identification of the points $(x, t) \in X \times \mathcal{T}$ with $t \in \mathcal{T} \backslash L \subset \mathbb{I}^{2}$ and the identification of each set $X \times\{t\}$ with the point $t$, for every $t \in L([3])$. 
Define the following closed subspace of $X \times \mathbb{I}^{2}$

$$
Y=X \times\{0\} \times \mathbb{I} \cup \bigcup_{n \in \mathbb{N}} X \times\{1 / n\} \times \mathbb{I} \cup\{x\} \times \mathbb{I} \times \mathbb{I} .
$$

The Alternating cone $A C(X, x)$ over a compact pointed space $(X, x)$ is defined as the quotient space of $Y$ via the identification of each set $X \times$ $\{0\} \times\{y\}$ to $(0 ; y), X \times\{1 /(2 n-1)\} \times\{0\}$ to $A_{2 n-1}=(1 /(2 n-1) ; 0)$ and $X \times\{1 / 2 n\} \times\{1\}$ to $B_{2 n}=(1 / 2 n ; 1)$ for each $y \in \mathbb{I}$ and each $n \in \mathbb{N}$, respectively.

In both cases of $S C(X, x)$ and $A C(X, x)$ let $p: S C(X, x) \rightarrow \mathbb{I}^{2}$ or $p:$ $A C(X, x) \rightarrow \mathbb{I}^{2}$ be the natural projection and define $p_{1}$ and $p_{2}$ by $p(u)=$ $\left(p_{1}(u) ; p_{2}(u)\right)$.

The spaces $C S C(X, x)$ and $C A C(X, x)$ are obtained from spaces $S C(X, x)$ and $A C(X, x)$, respectively by identifying each point $(a ; b) \in \mathbb{I}^{2}$ with $(0 ; b)$ for all $a, b \in \mathbb{I}$, i.e., by collapsing $\mathbb{I}^{2}$ to $L$. We also denote this distinguished interval by $L$. The projection $p_{2}$ is defined on all the spaces $S C(X, x), A C(X, x), C S C(X, x)$, and $C A C(X, x)$, while $p_{1}$ is defined only on $S C(X, x)$ and $A C(X, x)$.

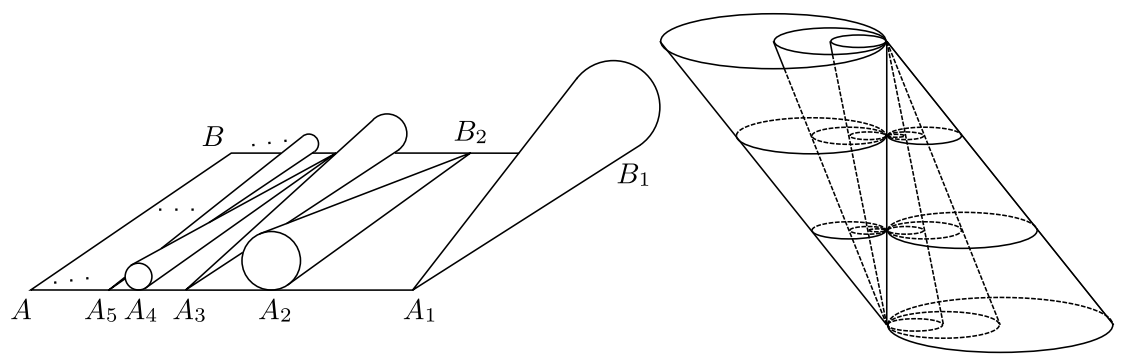

Figure 2. $A C\left(S^{1}\right)$ and $C A C\left(S^{1}\right)$

For $m, n \in \mathbb{N}$ with $m \leq n$, define $S C_{m, n}(X, x)=p_{1}^{-1}([1 / n, 1 / m])$ and $S C_{m}(X, x)=p_{1}^{-1}([0,1 / m])$ when $p$ is mapping from $S C(X, x)$ to $\mathbb{I}^{2}$, and define $A C_{m, n}(X, x)=p_{1}^{-1}([1 / n, 1 / m])$ and $A C_{m}(X, x)=p_{1}^{-1}([0,1 / m])$ when $p$ is mapping from $A C(X, x)$ to $\mathbb{I}^{2}$.

For a subspace $S$ of these spaces and a map $f$ defined on $S$ whose range is one of these spaces, $f$ is called flat, if $p_{2}(u)=p_{2}(v)$ implies $p_{2}(f(u))=$ $p_{2}(f(v))$ for $u, v \in S$. Similarly, a homotopy $H: S \times \mathbb{I} \rightarrow Z$ where $Z$ is one of these four spaces, is said to be flat, if the map $H(-, t)$ is flat for each $t \in \mathbb{I}$.

For a subspace $S$ of these spaces and a map $f$ defined on $S$ whose range is one of these spaces, $f$ is called level-preserving (resp., $\varepsilon$-level-preserving), if $p_{2}(f(u))=p_{2}(u)$ (resp., $\left|p_{2}(f(u))-p_{2}(u)\right|<\varepsilon$ ) for every $u \in S$. Similarly, a homotopy $H: S \times \mathbb{I} \rightarrow Z$ where $Z$ is one of these four spaces, is level-preserving 
(resp., $\varepsilon$-level-preserving), if $p_{2}(H(u), t)=p_{2}(u)$ (resp., $\left|p_{2}(H(u, t))-p_{2}(u)\right|<$ $\varepsilon$ ) for every $u \in S$ and $t \in \mathbb{I}$.

The cone over a space $X$, denoted by $C(X)$, is defined as the quotient space of $X \times \mathbb{I}$ by identifying $X \times\{1\}$ to a point and so we can describe points on the cone by points on $X \times \mathbb{I}$.

When arguments are not related to base points, we shall abbreviate $S C(X, x)$ by $S C(X)$, and so on.

Finally, we introduce a construction of spaces for our further investigation. For spaces $X_{n}(n \in \mathbb{N})$ with $x_{n} \in X_{n}$, let $\widetilde{\bigvee}_{n \in \mathbb{N}}\left(X_{n}, x_{n}\right)$ be the space obtained by identifying all $x_{n}$ 's to the point $x^{*}$ so that every neighborhood of $x^{*}$ contains almost all $X_{n}$ 's and each subspace topology of $X_{n}$ coincides with the topology of $X_{n}$. When the index set is finite, we write $\bigvee_{n=1}^{k}\left(X_{n}, x_{n}\right)$ as usual. When each $X_{n}$ is a copy of the circle, $\widetilde{V}_{n \in \mathbb{N}}\left(X_{n}, x_{n}\right)$ is homeomorphic to the Hawaiian earring. When each $X_{n}$ is a copy of the 2-dimensional torus $T$, we call $\widetilde{\bigvee}_{n \in \mathbb{N}}\left(X_{n}, x_{n}\right)$ the Hawaiian tori wedge and denote it by $\mathbb{H}_{T}$.

Remark 2.1. The Sombrero space was introduced and studied in [1]. The piecewise linear Sombrero space is defined as the subspace of $\mathbb{R}^{3}$ obtained by rotating $\mathcal{T}$ about its limiting interval $L \subseteq\{0\} \times\{0\} \times \mathbb{R}$. The Sliced Sombrero space is then defined as the union of the Sombrero space and $\mathbb{I} \times\{0\} \times \mathbb{I}$. In other words, the Sombrero space is the quotient space of the product $S^{1} \times \mathcal{T}$ that results from identifying the circles $S^{1} \times\{a\}$ for $a \in L \subset \mathcal{T}$ to one point, and the Sliced Sombrero space is the quotient space of the topological sum of the Sombrero space and the unit square $\mathbb{I}^{2}$ that results from identifying the two topological sine-curves $\mathcal{T}$ which have been defined in each of the components of this topological sum.

In this form one can see that the Sliced Sombrero and the Snake on a square $S C\left(S^{1}\right)$ are analogously built and thus are homeomorphic. Our starting point of the investigations in this paper was the discovery of this fact and the homotopy equivalence between the Sliced Sombrero and $A C\left(S^{1}\right)$, which is reflected in Theorem 1.2.

While describing the proof of Theorem 1.2, we found the constructions $C S C(X, x)$ and $C A C(X, x)$. Though differences among the constructions $S C(X, x), A C(X, x), C S C(X, x)$ and $C A C(X, x)$, except the difference between $C S C(X, x)$ and $C A C(X, x)$, were shown using the Hawaiian earring $\mathbb{H}$, the difference was left open. Similarly as for the circle $S^{1}, S C\left(S^{n}\right)$ and $A C\left(S^{n}\right)$ are homotopy equivalent for the $n$-sphere $S^{n}$, which was meanwhile shown by the first author. We found that $S C(T)$ and $A C(T)$ are not homotopy equivalent for the torus $T$ and by Theorem 1.1 $C S C(T)$ and $C A C(T)$ are not homotopy equivalent, either. Since a space which is homotopy equivalent to the Hawaiian tori wedge, appears in these spaces and it works in the proof, our interests turned to $H_{2}\left(\mathbb{H}_{T}\right)$. This explains how our results in this paper are related. 


\section{Proof of Theorems 1.1 And 1.2}

First we give another presentation of the space $C S C(X, x)$ and the space $C A C(X, x)$. Let $X_{n}$ be a copy of $X$ and $x_{n}$ a copy of $x_{0} \in X$ for each $n$. Then $C S C(X, x)$ is homeomorphic to the quotient space of $\widetilde{\bigvee}_{n \in \mathbb{N}}\left(X_{n}, x_{n}\right) \times$ $\mathbb{I}$, obtained by identifying $X_{2 n} \times\{0\}$ with $X_{2 n-1} \times\{0\}$, and by identifying $X_{2 n+1} \times\{1\}$ with $X_{2 n} \times\{1\}$ for $n \in \mathbb{N}$.

To present $C A C(X, x)$ in another way, let $C\left(\widetilde{\bigvee}_{n \in \mathbb{N}}\left(X_{2 n-1}, x_{2 n-1}\right)\right)$ be the cone over $\widetilde{\bigvee}_{n \in \mathbb{N}}\left(X_{2 n-1}, x_{2 n-1}\right)$, where $x^{*}$ is the point of identification and $C\left(\widetilde{\bigvee}_{n \in \mathbb{N}}\left(X_{2 n}, x_{2 n}\right)\right)$ with $x^{* *}$ analogously. We denote the interval connecting the vertex of the first cone and the base point $x^{*}$ by $\left\{x^{*}\right\} \times \mathbb{I}$, where $\left(x^{*}, 1\right)$ denotes the vertex and $\left(x^{*}, 0\right)$ denotes the point $x^{*}$ in the base space $\widetilde{V}_{n \in \mathbb{N}}\left(X_{2 n-1}, x_{2 n-1}\right)$. We denote the corresponding interval by $\left\{x^{* *}\right\} \times \mathbb{I}$, analogously for the second cone. Then $C A C\left(X, x_{0}\right)$ is homeomorphic to the quotient space of the disjoint union

$$
\left.C\left(\widetilde{\bigvee}_{n \in \mathbb{N}}\left(X_{2 n-1}, x_{2 n-1}\right)\right) \sqcup C \widetilde{\left(\widetilde{\bigvee}_{n \in \mathbb{N}}\right.}\left(X_{2 n}, x_{2 n}\right)\right)
$$

via the identification of $\left(x^{*}, t\right)$ with $\left(x^{* *}, 1-t\right)$ for $t \in \mathbb{I}$.

A space $X$ is called semi-locally strongly contractible at $x_{0} \in X$, if there exists a neighborhood $U$ of $x$ and a continuous map $H: U \times \mathbb{I} \rightarrow X$ such that $H(u, 1)=u, H(u, 0)=x_{0}$ and $H\left(x_{0}, t\right)=x_{0}$, for every $u \in U$ and $t \in \mathbb{I}$.

Proof of Theorem 1.1. By the semi-local strong contractibility at $x_{0} \in X$, we have $U$ and $H$ as above. Choose neighborhoods $V_{0}$ and $V_{1}$ of $x_{0}$ and continuous maps $F, G: X \rightarrow \mathbb{I}$ such that $\overline{V_{0}} \subseteq V_{1}, \overline{V_{1}} \subseteq U, F\left(x_{0}\right)=0$ and $F(x)=1$ for $x \notin V_{0}$ and $G(x)=0$ for $x \in \overline{V_{0}}$ and $G(x)=1$ for $x \notin V_{1}$. We denote the quotient map from $S C\left(X, x_{0}\right)$ to $C S C\left(X, x_{0}\right)$ by $\sigma$. Observe that the restriction of $\sigma$ to $p^{-1}(\mathcal{T}) \backslash \mathcal{T}$ is a bijection onto $C S C\left(X, x_{0}\right)$. We define $\tau: C S C\left(X, x_{0}\right) \rightarrow S C\left(X, x_{0}\right)$ by:

$$
\tau(\sigma(P, x))= \begin{cases}\left(F(x) \cdot p_{1}(P) ; p_{2}(P)\right), & \text { for } x \in V_{0} \\ (P, H(x, G(x))), & \text { for } x \in V_{1} \backslash V_{0} \\ (P, x), & \text { for } x \notin V_{1}\end{cases}
$$

for $P \in \mathcal{T}$ and $x \in X$. Since every element of $C S C\left(X, x_{0}\right)$ can be expressed as $\sigma(P, x)$ and $\left(F\left(x_{0}\right) \cdot p_{1}(P) ; p_{2}(P)\right)=\left(0 ; p_{2}(P)\right), \tau$ is well-defined. Points from copies of $V_{0}$ are mapped by $\tau$ into the square of $S C\left(X, x_{0}\right)$ and points from copies of $X \backslash V_{1}$ are mapped by $\tau$ into copies of $X$ in $S C\left(X, x_{0}\right)$. Therefore, using only level-preserving homotopies we can show that $\tau \circ \sigma$ is homotopic to $\operatorname{id}_{S C\left(X, x_{0}\right)}$ and that $\sigma \circ \tau$ is also homotopic to $\operatorname{id}_{C S C\left(X, x_{0}\right)}$.

We use the same maps $F, G$ and $H$, and also denote by $\sigma$ the quotient map from $A C\left(X, x_{0}\right)$ to $C A C\left(X, x_{0}\right)$. When we use the presentation of 
$C A C\left(X, x_{0}\right)$ via the cones

$$
C\left(\widetilde{\bigvee}_{n \in \mathbb{N}}\left(X_{2 n-1}, x_{2 n-1}\right)\right) \text { and } C\left(\widetilde{\bigvee}_{n \in \mathbb{N}}\left(X_{2 n}, x_{2 n}\right)\right),
$$

$V_{0, n}, V_{1, n}$ and $U_{n}$ are copies of $V_{0}, V_{1}$ and $U$ on $X_{n}$, respectively and we also use $F, G$ and $H$ for the copies.

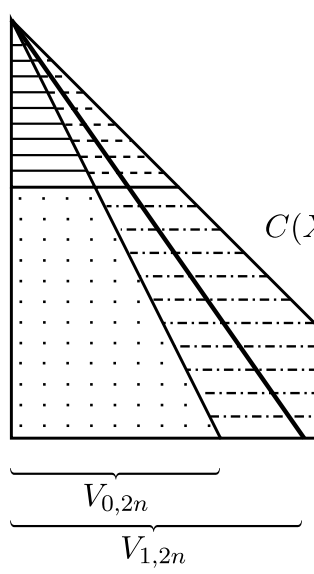

Figure 3. The figure visualizes the mapping $\tau$. The hatching styles indicate to which regions the corresponding domains are mapped. Observe that only $p \circ \tau$ is pictured; in particular the $\tau$-image of the dash-dotted area is considerably bigger than it is visible from the figure.

The homotopy equivalence between $A C\left(X, x_{0}\right)$ and $C A C\left(X, x_{0}\right)$ is proved similarly, but more care is necessary, because the homotopy inverse $\tau$ of $\sigma$, which we shall define in the sequel, is not level-preserving. We define $\tau$ only on $C\left(\widetilde{\bigvee}_{n \in \mathbb{N}}\left(X_{2 n}, x_{2 n}\right)\right)$ for notational convenience, but the other case is similar. Let $\tau(x, s)=$

$$
\left\{\begin{array}{lc}
((1-s) F(x) ; s+(1-s) F(x)) & \text { for }(2 n-1) /(2 n) \leq s \leq 1, x \in X_{2 n}, \\
(F(x) /(2 n) ; s(1+F(x) /(2 n-1))) & \text { for } 0 \leq s<(2 n-1) /(2 n), x \in V_{0,2 n}, \\
(H(x, G(x)), 2 n s /(2 n-1)) & \text { for } 0 \leq s<(2 n-1) /(2 n), \\
(x, 2 n s /(2 n-1)) & \quad x \in V_{1,2 n} \backslash V_{0,2 n}, \\
& \text { otherwise. }
\end{array}\right.
$$

Figure 3 shows the restriction of $p \circ \tau$ to $C\left(X_{2 n}\right)$. We remark that $\sigma(0 ; t)=$ $\left(x^{*}, t\right)$ and $\tau\left(x^{*}, t\right)=(0 ; t)$ and $(1-2 n) /(2 n)$ converge to 1 . Using this information we can see that $\tau \circ \sigma$ is homotopic to $\operatorname{id}_{A C\left(X, x_{0}\right)}$ and $\sigma \circ \tau$ is homotopic to $\operatorname{id}_{C A C\left(X, x_{0}\right)}$. 
Proof of TheOrem 1.2. By virtue of Theorem 1.1 the fact that $C S C\left(S^{1}\right)$ and $C A C\left(S^{1}\right)$ are homotopy equivalent implies the present theorem. To find this homotopy equivalence, we use embeddings of these spaces in $\mathbb{R}^{3}$. We define subsets of these embeddings as follows:

$$
\begin{aligned}
& S_{n}=\left\{(x, y, z) \mid(x-1 / n)^{2}+y^{2}=1 / n^{2}, z \in \mathbb{I}\right\}, \\
& T_{n}=\left\{(x, y, z) \mid\left(x-\left(\frac{1}{n+1}+\frac{z}{n(n+1)}\right)\right)^{2}+y^{2}\right. \\
& \left.=\left(\frac{1}{n+1}+\frac{z}{n(n+1)}\right)^{2}, z \in \mathbb{I}\right\}, \\
& U_{n}=\left\{(x, y, z) \mid(x-(1-z) / n)^{2}+y^{2}=(1-z)^{2} / n^{2}, z \in \mathbb{I}\right\} \text {, } \\
& V_{n}=\left\{(x, y, z) \mid(x+z / n)^{2}+y^{2}=z^{2} / n^{2}, z \in \mathbb{I}\right\},
\end{aligned}
$$

$S_{n}^{+}=S_{n} \cap(\mathbb{R} \times\{y \mid y \geq 0\} \times \mathbb{R})$ and $T_{n}^{+}=T_{n} \cap(\mathbb{R} \times\{y \mid y \geq 0\} \times \mathbb{R})$.

By the preceding description, $C S C\left(S^{1}\right)$ is homotopy equivalent to the subspace $Y_{0}=\bigcup_{n \in \mathbb{N}}\left(S_{n} \cup T_{n}\right)$ of $\mathbb{R}^{3}$. Let $Y_{1}$ be the following subspace of $\mathbb{R}^{3}$ (see Figure 4$)$ :

$$
[0,2] \times\{0\} \times \mathbb{I} \cup \bigcup_{n \in \mathbb{N}} S_{n}^{+} \cup T_{n}^{+}
$$
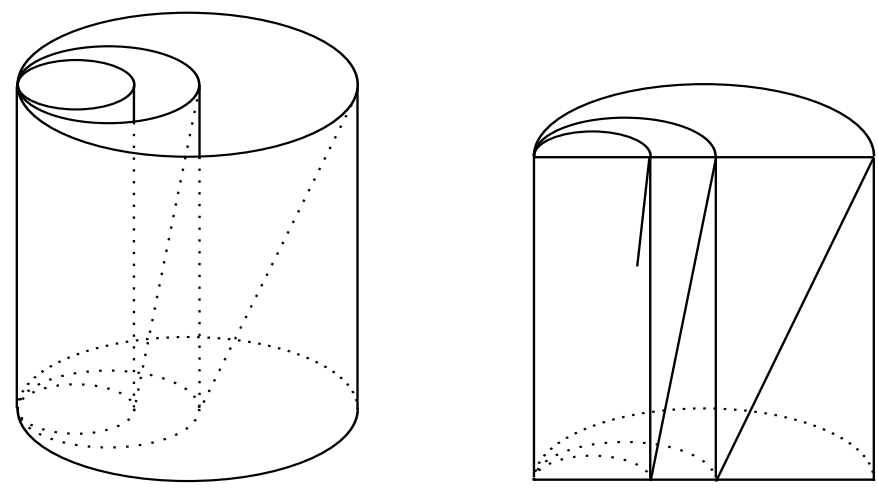

Figure 4. $C S C\left(S^{1}\right)$ and $Y_{1}$

The homotopy equivalence between the spaces $\bigcup_{n \in \mathbb{N}}\left\{(x, y) \mid(x-1 / n)^{2}+\right.$ $\left.y^{2}=1 / n^{2}\right\}$ and $[0,2] \times\{0\} \cup \bigcup_{n \in \mathbb{N}}\left\{(x, y) \mid(x-1 / n)^{2}+y^{2}=1 / n^{2}, y \geq 0\right\}$ induces a homotopy equivalence between $Y_{0}$ and $Y_{1}$ and it now suffices to establish the homotopy equivalence between $Y_{1}$ and $C A C\left(S^{1}\right)$. We use the presentation of $C A C\left(S^{1}\right)$ from the introduction of Section 3 and we realize it 
ON SNAKE CONES, ALTERNATING CONES AND RELATED CONSTRUCTIONS 123 in $\mathbb{R}^{3}$ as the following subspace:

$$
\bigcup_{n \in \mathbb{N}} U_{n} \cup V_{n}
$$

Define $\sigma: Y_{1} \rightarrow C A C\left(S^{1}\right)$ so that $\sigma(x, y, z)=(0,0, z)$, if

$$
\left(x-\frac{1}{n}\right)^{2}+y^{2}=\left(\frac{1}{n}\right)^{2}
$$

or if

$$
\left(x-\left(\frac{1}{n+1}+\frac{z}{n(n+1)}\right)\right)^{2}+y^{2}=\left(\frac{1}{n+1}+\frac{z}{n(n+1)}\right)^{2}
$$

for $n \in \mathbb{N}$ and $z \in \mathbb{I}$, and so that $\sigma$ maps level-preserving from

$$
\left\{(x ; y) \mid 0 \leq y \leq \frac{x-1 /(n+1)}{n(n+1)}, \frac{1}{n+1} \leq x \leq \frac{1}{n}\right\}
$$

onto $U_{n}$ and from

$$
\left\{(x ; y) \mid \frac{x-1 /(n+1)}{n(n+1)} \leq y \leq 1, \frac{1}{n+1} \leq x \leq \frac{1}{n}\right\}
$$

onto $V_{n}$.

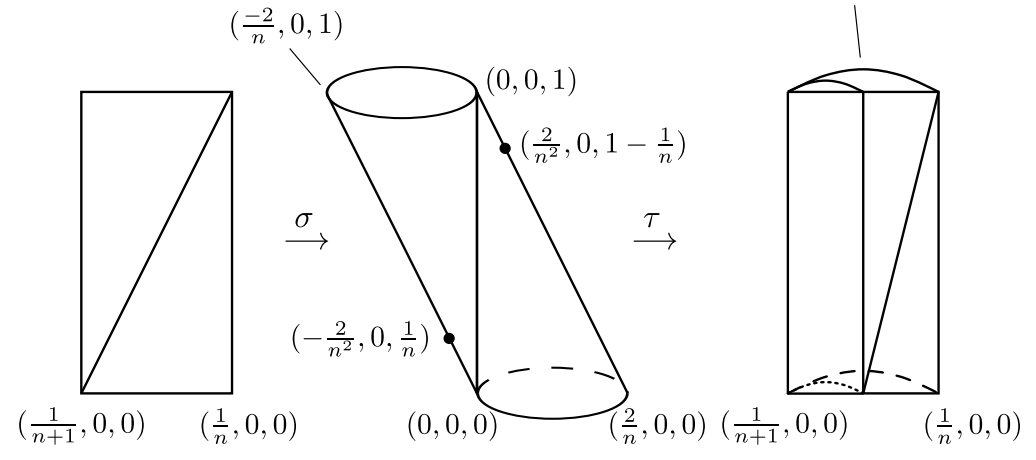

Figure 5. Partial illustrations of the maps $\sigma$ and $\tau$.

We define $\tau: Y_{1} \rightarrow C A C\left(S^{1}\right)$ so that $\tau(0,0, z)=(0,0, z)$ for $z \in \mathbb{I}$ and so that $\tau$ maps $U_{n}$ homeomorphically onto

$$
S_{n}^{+} \cup T_{n}^{+} \cup\left\{(x, y, z) \mid \frac{1}{n+1} \leq x \leq 1 / n, y=0,0 \leq z \leq \frac{x-1 /(n+1)}{n(n+1)}\right\}
$$


and $V_{n}$ onto

$$
S_{n+1}^{+} \cup T_{n}^{+} \cup\left\{(x, y, z) \mid \frac{1}{n+1} \leq x \leq 1 / n, y=0, \frac{x-1 /(n+1)}{n(n+1)} \leq z \leq 1\right\} .
$$

We define $\tau$ uniformly with respect to $n$, in particular so that $\tau$ is $(1 / n)$-levelpreserving on $U_{n}$ and $V_{n}$.

In particular, $\tau$ maps the segment $\left[(0,0,1),\left(2 / n^{2}, 1-1 / n\right)\right]$ onto the arc

$$
\left\{(x, y, 1) \mid(x-1 / n)^{2}+y^{2}=1 / n^{2}, y \geq 0\right\}
$$

and the segment $\left[(0,0,0),\left(-2 / n^{2}, 0,1 / n\right)\right]$ onto the arc

$$
\left\{(x, y, 0) \mid(x-1 /(n+1))^{2}+y^{2}=1 /(n+1)^{2}, y \geq 0\right\}
$$

which is the only dotted arc that we have drawn in Figure 5.

Since the segments $\left[(0,0,1),\left(2 / n^{2}, 0,1-1 / n\right)\right]$ converge to $(0,0,1)$ and $\left[(0,0,0),\left(-2 / n^{2}, 0,1 / n\right)\right]$ converge to $(0,0,0)$ when $n$ tends to infinity and $\sigma$ is level-preserving, and $\tau$ is $(1 / n)$-level-preserving on $U_{n}$ and $V_{n}$, we can conclude that $\sigma \circ \tau$ and $\tau \circ \sigma$ are homotopic to the identity mapping on $C A C\left(S^{1}\right)$ and $Y_{1}$, respectively.

\section{Proof of Theorem 1.3}

The first lemma concerns a certain useful property of $A C(X, x)$ for any path-connected space $X$.

Lemma 4.1. Let $X$ be a path-connected space. Then $p_{2}^{-1}([0,1))$ and $p_{2}^{-1}((0,1])$ are contractible in $A C(X, x)$. The same also holds for $C A C(X, x)$.

Proof. Since $p_{2}^{-1}(\{0\})$ is a strong deformation retract of $p_{2}^{-1}([0,1))$, it suffices to show that $p_{2}^{-1}(\{0\})$ is contractible in $A C(X, x)$. Since $p_{2}^{-1}(\{0\})$ deforms to the segment $[(0 ; 1),(1 ; 1)], p_{2}^{-1}(\{0\})$ is contractible in $A C(X, x)$. The contractibility of $p_{2}^{-1}((0,1])$ in $A C(X, x)$ can be proved similarly. In the case of $C A C(X, x)$ the above procedure yields a point instead of the segment $[(0 ; 0),(1 ; 0)]$.

We prove (1),(2) and (3) separately.

\subsection{Proof of Theorem 1.3: (1) and (2).}

Proof of Theorem $1.3(1)$. The fact that $S C(\mathbb{H}, o)$ and $A C(\mathbb{H}, o)$ are not homotopy equivalent was proved in [7, Theorem 3.6], where it was established that any embedding of $\mathbb{H}$ to $A C(\mathbb{H}, o)$ is null homotopic, while there exists an essential embedding of $\mathbb{H}$ to $S C(\mathbb{H}, o)$.

Hence, for the second nonequivalence it suffices to show that any embedding of $\mathbb{H}$ to $C S C(\mathbb{H}, o)$ is null homotopic. Let $f: \mathbb{H} \rightarrow C S C(\mathbb{H}, o)$ be an embedding. Let $x^{*}$ be the identified points with copies of $o \in \mathbb{H}$. Since $C S C(\mathbb{H}, o)$ is locally contractible at points in $C S C(\mathbb{H}, o) \backslash\left\{x^{*}\right\} \times \mathbb{I}$, $f(o)$ belongs to $\left\{x^{*}\right\} \times \mathbb{I}$, which is the crucial difference between $C S C(\mathbb{H}, o)$ 
and $S C(\mathbb{H}, o)$. Therefore, except for finitely many circles of $\mathbb{H}, f$ maps into $p_{2}^{-1}([0,1))$ or $p_{2}^{-1}((0,1])$. By a similar argument as in the proof of $[3$, Lemma $3.3]$ we can show that $f$ is homotopic to a map $f^{\prime}$ into $p_{2}^{-1}([0,1))$ or $p_{2}^{-1}((0,1])$. By a similar argument as in the proof of [3, Theorem 1.1] we can then produce a null-homotopy of $f^{\prime}$.

For a proof of Theorem 1.3(2) we need some notions and lemmas. For $A C(Z)$ and $P \in \bigcup_{n \in \mathbb{N}}\{1 /(2 n-1)\} \times(0,1] \cup\{1 /(2 n) \times[0,1)\}$, let $Z_{P}=$ $p^{-1}(\{P\})$, which is homeomorphic to $Z$ and $M_{s}=p_{2}^{-1}(\{s\})$ for $s \in \mathbb{I}$.

An interval $[a, b]$ with $0 \leq a<b \leq 1$ is called an essential interval for a flat continuous map $f: A C_{n}(Z) \rightarrow A C(Z)$, if the following hold:

(1) $f$ maps $p_{2}^{-1}(\{a\}) \cap A C_{n}(Z)$ into $M_{0}$ and $p_{2}^{-1}(\{b\})$ into $M_{1}$;

(2) $f$ maps $p_{2}^{-1}((a, b)) \cap A C_{n}(Z)$ into $p_{2}^{-1}((0,1))$; and

(3) for every $a<c<b, f \mid p_{2}^{-1}\{c\}$ is homotopic to the restriction of the identity mapping on $A C_{n}(Z)$ to $p_{2}^{-1}\{c\}$ in $p_{2}^{-1}((0,1))$.

For $s \in(0,1)$ and $t \in \mathbb{I}$, we define a property $P(s, t)$ of $H$ as follows:

$H\left(M_{s} \cap A C_{n}(Z), t\right) \subseteq p_{2}^{-1}((0,1))$ and the restriction of $H(-, t)$ to $M_{s} \cap A C_{n}(Z)$ is homotopic to the identity mapping on $M_{s} \cap$ $A C_{n}(Z)$ in $p_{2}^{-1}((0,1))$.

We remark that by the flatness of $H$, if $H\left(M_{s} \cap A C_{n}(Z), t\right) \subseteq p_{2}^{-1}((0,1))$, there is a neighborhood $U$ of $(s ; t)$ such that $H\left(M_{s^{\prime}} \cap A C_{n}(Z), t^{\prime}\right) \subseteq p_{2}^{-1}((0,1))$ for any $\left(s^{\prime} ; t^{\prime}\right) \in U$.

LEMMA 4.2. Let $Z$ be a compact path-connected space and $n \in \mathbb{N}$. Let $f: A C_{n}(Z) \rightarrow A C(Z)$ (or $f: A C_{n}(Z) \rightarrow C A C(Z)$ ) be a continuous map. If for each $y \in \mathbb{I}$ the set $p_{2}\left(f\left(M_{y} \cap A C_{n}(Z)\right)\right)$ does not contain both points 0 and $1, f$ is homotopic to a flat map $f_{1}$.

Moreover, let $H: A C_{n}(Z) \times \mathbb{I} \rightarrow A C(Z)$ be a homotopy between the identity mapping on $A C_{n}(Z)$ and $f$ such that for each $y \in \mathbb{I}$ and $t \in \mathbb{I}$, the set $p_{2}\left(H\left(M_{y} \cap A C_{n}(Z), t\right)\right)$ does not contain both points 0 and 1 . Then there exists a flat homotopy between the identity mapping and $f_{1}$.

Proof. Fix the number $y$. Let $A(y)$ and $B(y)$ be the minimum and the maximum of the function $p_{2} \circ f: M_{y} \cap A C_{n}(Z) \rightarrow \mathbb{I}$, respectively, and also let

$$
C(y)=\frac{A(y)}{1+A(y)-B(y)} .
$$

We remark that the continuity of $A(y)$ and $B(y)$ follows from the fact that the shape of $A C_{n}(Z)$ is like a direct product.

Let $\varphi\left(s_{0}, s_{1}, t\right)=s_{0}+\left(s_{1}-s_{0}\right) t$. For $x \in p_{2}^{-1}((0,1))$ and $u \in \mathbb{I}$, let $x_{u} \in p_{2}^{-1}(\{u\})$ be the point determined by the retraction of $p_{2}^{-1}\left(\left[p_{2}(x), u\right]\right)$ or $p_{2}^{-1}\left(\left[u, p_{2}(x)\right]\right)$ to $p_{2}^{-1}(\{u\})$, which is derived from the direct product structure, 
and for $x \in p_{2}^{-1}(\{0\} \cup\{1\})$ and $u \in \mathbb{I}$, let $x_{u}=x$. Define $F: A C_{n} \times \mathbb{I} \rightarrow A C_{n}$ by

$$
F(x, t)=x_{\varphi\left(p_{2}(f(x)), C\left(p_{2}(f(x))\right), t\right)} .
$$

Since $A\left(p_{2}(f(x)), t\right)=0$ implies $C\left(p_{2}(f(x)), t\right)=0$ and $B\left(p_{2}(f(x)), t\right)=1$ implies $C\left(p_{2}(f(x))\right)=1$ and both cannot occur at the same time, $F$ is a deformation retraction, $F(-, 0)$ is $f$ and $F(-, 1)$ is a flat map.

If $f$ is a flat map, then $f_{1}=f$. Since the identity mapping on $A C_{n}$ is a flat map, applying this reasoning to the homotopy $H$ we have a homotopy $\bar{H}$ for the second statement, i.e., we define $A(y, t), B(y, t), C(y, t)$ using $H(x, t)$ instead of $f(x)$ and let

$$
\bar{H}(x, t)=H(x, t)_{\varphi\left(p_{2}(H(x, t)), C\left(p_{2}(H(x, t), t)\right), t\right)} .
$$

LEMMA 4.3. Let $Z$ be a noncontractible space and $H: A C_{2 m-1,2 m}(Z) \times$ $\mathbb{I} \rightarrow A C(Z)$ a flat homotopy. If $H\left(M_{0} \cap A C_{2 m-1,2 m}(Z), t_{0}\right) \subseteq p_{2}^{-1}((0,1))$, then there exists a neighborhood $U$ of $\left(0 ; t_{0}\right)$ such that $H$ does not satisfy $P(s, t)$ for any $(s ; t) \in U$ with $s>0$. An analogous statement holds for $H\left(M_{1} \cap A C_{2 m-1,2 m}(Z), t_{0}\right) \subseteq p_{2}^{-1}((0,1))$.

Proof. We have a neighborhood $U$ of $\left(0 ; t_{0}\right)$ such that

$$
H\left(M_{s} \cap A C_{2 m-1,2 m}, t\right) \subseteq p_{2}^{-1}((0,1))
$$

for any $(s ; t) \in U$. We fix $(s ; t) \in U$ with $s>0$. Let $P_{n}=(1 / n ; s)$ and $I_{n}=\left[P_{n+1}, P_{n}\right]$. Then we have $M_{s}=\{(0 ; s)\} \cup \bigcup_{n=1}^{\infty} I_{n} \cup Z_{P_{n}}$ and $M_{s} \cap A C_{2 m-1,2 m}(Z)=Z_{P_{2 m}} \cup Z_{P_{2 m-1}} \cup I_{2 m-1}$. Since $H(-, t)$ maps $\bigcup_{u \in\left[P_{2 m-1}, A_{2 m-1}\right]} Z_{u}$ into $p^{-1}(\mathbb{I} \times(0,1))$, the restriction of $H(-, t)$ to $Z_{P_{2 m-1}}$ is null-homotopic in $p^{-1}(\mathbb{I} \times(0,1))$. Since $M_{s}$ is a strong deformation retract of $p^{-1}(\mathbb{I} \times(0,1))$ and $Z_{P_{2 m-1}}$ is a retract of $p^{-1}(\mathbb{I} \times(0,1))$ and $Z_{P_{2 m-1}}$ is not contractible, the identity mapping on $Z_{P_{2 m-1}}$ is not homotopic to the restriction of $H(-, t)$ to $Z_{P_{2 m-1}}$, which implies that $P(s, t)$ does not hold.

To prove the statement for $H\left(M_{1}, t_{0}\right)$ we use $Z_{P_{2 m}}$ and argue on a neighborhood of $B_{2 m}$ to obtain a similar conclusion.

Lemma 4.4. Let $H: A C_{n}(\mathbb{H}, o) \times \mathbb{I} \rightarrow A C(\mathbb{H}, o)$ be a flat homotopy between the identity mapping on $A C_{n}(\mathbb{H}, o)$ and $f$. Then there exist $a_{0}, b_{0} \in \mathbb{I}$ with $a_{0}<b_{0}$ such that $\left[a_{0}, b_{0}\right]$ is an essential interval for $f$.

Proof. Let $d:[0,1] \rightarrow S^{1}$ be a winding with the base point $z_{0}$, i.e., both $d \mid[0,1)$ and $d \mid(0,1]$ are bijective continuous mappings with $d(0)=d(1)=z_{0}$.

We define a homotopy $H^{*}: S^{1} \times \mathbb{I} \rightarrow S^{1}$ as follows:

$$
H^{*}(u, t)=\left\{\begin{aligned}
d\left(p_{2}\left(H\left(M_{d^{-1}(u)}, t\right)\right)\right) & \text { if } u \neq z_{0} \text { and } P\left(d^{-1}(u), t\right) \text { holds; } \\
z_{0}, & \text { otherwise. }
\end{aligned}\right.
$$

First we show the continuity of $H^{*}$. 
If $u \neq z_{0}$ and $P\left(d^{-1}(u), t\right)$ holds, the continuity at $(u, t)$ is clear. Otherwise, $u \neq z_{0}$ and $P\left(d^{-1}(u), t\right)$ does not hold, or $u=z_{0}$. We consider two cases:

CASE 1. Suppose that $u \neq z_{0}$ and $P\left(d^{-1}(u), t\right)$ does not hold: If $p_{2} \circ$ $H\left(M_{d^{-1}(u)}, t\right)=\{0\}$ or $\{1\}$, then the continuity at $(u, t)$ follows from that of $H$. Otherwise, since $H(-, t)$ maps $M_{d^{-1}(u)} \cap A C_{n}(Z)$ continuously with respect to $u$ and $t$, the restriction of $H(-, t)$ to $M_{d^{-1}(u)} \cap A C_{n}(Z)$ is not homotopic to the identity mapping on $M_{d^{-1}(u)} \cap A C_{n}(Z)$ in $p^{-1}(\mathbb{I} \times(0,1))$, i.e., $H^{*}$ takes the value $z_{0}$ in a neighborhood of $(u, t)$.

CASE 2. Suppose that $u=z_{0}$ : If each of $p_{2}\left(H\left(M_{0} \cap A C_{n}(Z), t\right)\right)$ and $p_{2}\left(H\left(M_{1} \cap A C_{n}(Z), t\right)\right)$ is equal to either $\{0\}$ or $\{1\}$, the continuity at $(u, t)$ follows from that of $H$. The remaining case is when $p_{2}\left(H\left(M_{0} \cap A C_{n}(Z), t\right)\right) \subseteq$ $(0,1)$ or $p_{2}\left(H\left(M_{1} \cap A C_{n}(Z), t\right)\right) \subseteq(0,1)$. In this case the continuity follows by Lemma 4.3 .

We have shown that $H^{*}$ is a homotopy and hence we have $a_{0}, b_{0} \in \mathbb{I}$ with $a_{0}<b_{0}$ such that $H^{*}\left(a_{0}, 1\right)=H^{*}\left(b_{0}, 1\right)=z_{0}, H^{*}(s, 1) \neq z_{0}$ for $a_{0}<s<b_{0}$ and the orientation of the winding of $H^{*}(-, 1)$ on $\left[a_{0}, b_{0}\right]$ is the same as that of $d$. The last statement implies that $f\left(a_{0}\right)=H\left(a_{0}, 1\right)=0$ and $f\left(b_{0}\right)=$ $H\left(b_{0}, 1\right)=1$. We have $P(s, 1)$ for $a_{0}<s<b_{0}$, which implies that $\left[a_{0}, b_{0}\right]$ is an essential interval for $f$.

Proof of TheOrem 1.3 (2). To show the nonequivalence by contradiction suppose that we have $f: A C(\mathbb{H}, o) \rightarrow C A C(\mathbb{H}, o)$ and $g: C A C(\mathbb{H}, o) \rightarrow$ $A C(\mathbb{H}, o)$ such that $g \circ f$ is homotopic to the identity mapping on $A C(\mathbb{H}, o)$ via a homotopy $H$.

There exists a sufficiently large $n$ such that the restriction of $H$ to $A C_{n}(\mathbb{H}, o) \times \mathbb{I}$ satisfies the condition for $H$ in Lemma 4.2. Apply Lemma 4.2 to the homotopy $H$ between the identity mapping on $A C_{n}(\mathbb{H}, o)$ and $g \circ$ $f \mid A C_{n}(\mathbb{H}, o)$, to get a flat homotopy $\bar{H}$ between the identity mapping on $A C_{n}(\mathbb{H}, o)$ and a map which is given by applying Lemma 4.2 and homotopic to $g \circ f \mid A C_{n}(\mathbb{H}, o)$. According to Lemma 4.4, we have an essential interval $\left[a_{0}, b_{0}\right]$ for $\bar{H}(-, 1)$. We remark that $g \circ f\left(p_{2}^{-1}\left(\left(a_{0}, b_{0}\right)\right) \cap A C_{n}(\mathbb{H}, o)\right) \subseteq p_{2}^{-1}((0,1))$.

First we claim that $f((1 / m ; s)) \in L$ for $a_{0} \leq s \leq b_{0}$ and $m \geq n$. To show this by contradiction, suppose that $f\left(\left(1 / m ; s_{0}\right)\right) \notin L$. Then $f\left(\left(1 / m ; s_{0}\right)\right)$ is contained in a local disk. This implies that the restriction of $\bar{H}(-, 1)$ to $p_{2}^{-1}\left(\left\{s_{0}\right\}\right)$ is not homotopic to the identity on $p_{2}^{-1}\left(\left\{s_{0}\right\}\right)$, since $\bar{H}(-, 1)$ is obtained from $g \circ f$ as in Lemma 4.2. Hence $f\left(\{1 / m\} \times\left[a_{0}, b_{0}\right]\right) \subseteq L$ for $m \geq n$. Thus, for sufficiently large distinct $m_{0}, m_{1}, f\left(\left\{1 / m_{0}\right\} \times\left[a_{0}, b_{0}\right]\right)$ and $f\left(\left\{1 / m_{1}\right\} \times\left[a_{0}, b_{0}\right]\right)$ are included as intervals on $L$. Therefore we conclude that some subinterval of $\left\{1 / m_{0}\right\} \times\left[a_{0}, b_{0}\right]$ or $\left\{1 / m_{1}\right\} \times\left[a_{0}, b_{0}\right]$ is mapped by $g \circ f$ outside of $\left\{1 / m_{0}\right\} \times(0,1)$ or outside of $\left\{1 / m_{1}\right\} \times(0,1)$, i.e., at least one of these intervals goes through the base square $\mathbb{I}^{2}$ by the homotopy $\bar{H}$. Hence 
the restriction of $\bar{H}(-, 1)$ to $M_{s} \cap A C_{n}(Z)$ is not homotopic to the identity on $p_{2}^{-1}((0,1))$ for $a_{0}<s<b_{0}$, which is a contradiction.

4.2. Proof of Theorem 1.3 (3). Next we show that $C S C(\mathbb{H}, o)$ and $C A C(\mathbb{H}, o)$ are homotopy equivalent. Actually we show that $C S C(\mathbb{H}, o)$ is homotopy equivalent to $C S C\left(S^{1}, z_{0}\right)$ and $C A C(\mathbb{H}, o)$ is homeomorphic to $C A C\left(S^{1}, z_{0}\right)$. Then the conclusion follows from Theorems 1.1 and 1.2. To describe $C S C(\mathbb{H}, o)$ and $C A C(\mathbb{H}, o)$, we introduce the constructions $\widetilde{\mathbb{I}}_{n \in \mathbb{N}} C S C\left(X_{n}, x_{n}\right)$ and $\widetilde{\mathbb{I}}_{n \in \mathbb{N}} C A C\left(X_{n}, x_{n}\right)$

For spaces $X_{n}$ with $x_{n} \in X_{n}$, let $Y_{n}=C S C\left(X_{n}, x_{n}\right)$ and $Z_{n}=$ $C A C\left(X_{n}, x_{n}\right)$. First we describe the construction of $\widetilde{\mathbb{I}}_{n \in \mathbb{N}} C S C\left(X_{n}, x_{n}\right)$. We identify all copies of $(0 ; s)$ in $Y_{n}$ for each $s \in \mathbb{I}$ and have the quotient set of the disjoint union of $Y_{n}$ 's. Then we induce a topology so that any neighborhood of $\{(0 ; s) \mid s \in \mathbb{I}\}$ contains almost all $Y_{n}$ 's. More precisely, let ${ }_{n} p$ and ${ }_{n} p_{2}$ be copies of the projections $p$ and $p_{2}$ for $Y_{n}$, respectively. Then, a basic neighborhood base of $(0 ; y)$ is

$$
\bigcup_{n=1}^{k}{ }_{n} p^{-1}([0, \varepsilon) \times(a, b)) \cup \bigcup_{n=k}^{\infty}{ }_{n} p_{2}^{-1}((a, b))
$$

for $\varepsilon>0$ and $a<y<b$. We denote this space by $\widetilde{\mathbb{I}}_{n \in \mathbb{N}} C S C\left(X_{n}, x_{n}\right)$.

We define $\widetilde{\mathbb{I}}_{n \in \mathbb{N}} C A C\left(X_{n}, x_{n}\right)$ analogously, i.e., we identify all copies of $(0 ; s)$ in $Z_{n}$ for each $s \in \mathbb{I}$ and have the quotient set of the disjoint union of $Z_{n}$ 's. Then we induce a topology so that any neighborhood of $\{(0 ; s) \mid s \in \mathbb{I}\}$ contains almost all $Z_{n}$ 's.

It is then easy to see that $C S C\left(\left(\widetilde{\mathrm{V}}_{n \in \mathbb{N}}\left(X_{n}, x_{n}\right), x^{*}\right)\right.$ is homeomorphic to $\widetilde{\mathbb{I}}_{n \in \mathbb{N}} C S C\left(X_{n}, x_{n}\right)$ and $C A C\left(\left(\widetilde{\bigvee}_{n \in \mathbb{N}}\left(X_{n}, x_{n}\right), x^{*}\right)\right.$ is homeomorphic to $\widetilde{\mathbb{I}}_{n \in \mathbb{N}} C A C\left(X_{n}, x_{n}\right)$, where $x^{*}$ denotes the point that results from the identification of all $x_{n}$.

Proof of TheOrem 1.3 (3). We recall the proof of Theorem 1.2. There, the maps except $\tau$ assuring homotopy equivalences are level-preserving. Moreover $\tau$ can be taken to be $\varepsilon$-level-preserving for each $\varepsilon>0$. Let $X_{n}$ be copies of the circle $S^{1}$. Accordingly, we have continuous maps $f_{n}: C S C\left(X_{n}\right) \rightarrow C A C\left(X_{n}\right), g_{n}: C A C\left(X_{n}\right) \rightarrow C S C\left(X_{n}\right)$ and homotopies $F_{n}: C S C\left(X_{n}\right) \times \mathbb{I} \rightarrow C S C\left(X_{n}\right), G_{n}: C A C\left(X_{n}\right) \times \mathbb{I} \rightarrow C A C\left(X_{n}\right)$ such that:

(1) $f_{n}$ is level-preserving;

(2) $g_{n}, F_{n}, G_{n}$ are $1 / n$-level-preserving; and

(3) $F_{n}((0 ; y), t)=(0 ; y)$ and $G_{n}((0 ; y), t)=(0 ; y)$ for $y, t \in \mathbb{I}$.

Define $f: \widetilde{\mathbb{I}}_{n \in \mathbb{N}} C S C\left(X_{n}\right) \rightarrow \widetilde{\mathbb{I}}_{n \in \mathbb{N}} C A C\left(X_{n}\right), \quad g: \widetilde{\mathbb{I}}_{n \in \mathbb{N}} C A C\left(X_{n}\right) \rightarrow$ $\widetilde{\mathbb{I}}_{n \in \mathbb{N}} C S C\left(X_{n}\right)$ and homotopies $F: \widetilde{\mathbb{I}}_{n \in \mathbb{N}} C S C\left(X_{n}\right) \times \mathbb{I} \rightarrow \widetilde{\mathbb{I}}_{n \in \mathbb{N}} C S C\left(X_{n}\right)$, $G: \widetilde{\mathbb{I}}_{n \in \mathbb{N}} C A C\left(X_{n}\right) \times \mathbb{I} \rightarrow \widetilde{\mathbb{I}}_{n \in \mathbb{N}} C A C\left(X_{n}\right)$ as unions of $f_{n}, g_{n}, F_{n}, G_{n}$ 
respectively. It suffices to verify their continuity. We show this for $F$, since the proofs for the others are similar. Continuity at points other than $(0 ; y)$ is obvious. For $\bigcup_{m=1}^{k_{0}} m p^{-1}([0, \varepsilon) \times(a, b)) \cup \bigcup_{m=k_{0} m}^{\infty} p_{2}^{-1}((a, b))$ with $\varepsilon>0$ and $a<y_{0}<b$, choose $k \geq k_{0}$ so that $a+1 / k<y_{0}<b-1 / k$. Then, we have $a<p_{2} \circ F_{m}((0 ; y), t)<b$ for $m \geq k$ and $t \in \mathbb{I}$. Hence using the continuity of $F_{m}$ for $m<k$ we get the desired neighborhood of $\left(0 ; y_{0}\right)$ which assures the continuity of $F$ at $\left(0 ; y_{0}\right)$. Since $(\mathbb{H}, o)$ is $\left(\widetilde{\mathrm{V}}_{n \in \mathbb{N}}\left(X_{n}, x_{n}\right), x^{*}\right)$, we conclude that $C S C(\mathbb{H}, o)$ and $C A C(\mathbb{H}, o)$ are homotopy equivalent.

\section{Proof of Theorem 1.4}

We shall consider 2-cycles in $S C(T, x)$, where $T$ is a 2-dimensional torus with a distinguished point $x$. For this purpose we prove some basic results about oriented closed surfaces and wedges of tori. Let $\left(T_{i}, x_{i}\right)$ be copies of the torus $(T, x)$ and $r_{i}: \bigvee_{i=1}^{n}\left(T_{i}, x_{i}\right) \rightarrow T_{i}$ be the retractions, where the attaching point of the wedge $\bigvee_{i=1}^{n}\left(T_{i}, x_{i}\right)$ is denoted by $o$ and $r_{i}$ maps $T_{j}$ to $o$ for $j \neq i$. We denote the genus of an oriented closed surface $S$ by $g(S)$. Let $z$ be a singular 2-cycle of a space $X$. We can write $z$ as a formal sum $\sum_{i=0}^{m} \mu_{i} g_{i}$ where $\mu_{i}= \pm 1$ and $g_{i}$ are continuous maps of the 2-simplex $\Delta_{2}$ to $X$. For a 2-cycle $z,[z]$ denotes the homology class containing $z$.

Since $z$ is a 2-cycle, by patching boundaries of copies of $\Delta_{2}$ we get an oriented closed surface $S_{z}$ and a continuous map $f_{z}: S_{z} \rightarrow X$. Let $\left[S_{z}\right]$ be the homology class of the fundamental cycle of $S_{z}$. Then we have $f_{z *}\left(\left[S_{z}\right]\right)=[z]$. We refer the reader to $[9$, pp.108-109] for this standard construction.

In case $S_{z}$ is not connected, we have 2-cycles $z_{1}, \cdots, z_{k}$ such that $\sum_{i=1}^{k} z_{i}=$ $z$, each $S_{z_{i}}$ is connected, and $S_{z}$ is the disjoint union of $S_{z_{i}}$ 's. An oriented closed surface $S_{z}$ constructed from a 2-cycle $z$ is generally not unique, but the following results hold for any construction of $S_{z}$.

Lemma 5.1. Let $z$ be a singular 2-cycle of $\bigvee_{i=1}^{n}\left(T_{i}, x_{i}\right)$. Then the cardinality of $\left\{i \mid r_{i *}([z]) \neq 0\right\}$ is at most $g\left(S_{z}\right)$.

Proof. We prove this by induction on the genus $g\left(S_{z}\right)$. By the preceding remark we may suppose that $S_{z}$ is connected. When $g\left(S_{z}\right)=0, S_{z}$ is a 2 -sphere and so $r_{i} \circ f_{z}$ is null-homotopic for each $i$ hence the conclusion is obvious. To prove the induction step by contradiction, suppose to the contrary. Without loss of generality we may assume that $g\left(S_{z}\right) \geq 1$ and $r_{i *}(z) \neq 0$ for $1 \leq i \leq g\left(S_{z}\right)+1$. We may also assume that $f_{z}$ is a piecewise linear map and $f_{z}^{-1}(\{o\})$ is a surface with boundary, by thickening if necessary.

We claim that we have a simple closed curve in $f_{z}^{-1}(\{o\})$ which is essential on $S_{z}$. To show this by contradiction, suppose that $f_{z}^{-1}(\{o\})$ does not contain any closed curve which is essential on $S_{z}$. Let $C$ be a connected component of $f_{z}^{-1}(\{o\})$. We have at most finitely many disjoint connected boundaries of $C$ which are simple closed curves. Since these are inessential, at least one 
side of this simple closed curve on $S_{z}$ is a disk. If this disk contains $C$ for at least two simple closed curves in the boundary of $C, S_{z}$ is a 2-sphere, which contradicts $g\left(S_{z}\right) \geq 1$. On the other hand, if this side does not contain $C$ for every simple closed curve in the boundary of $C, f_{z}$ is null-homotopic in this case, because $\pi_{2}\left(T_{i}\right)$ is trivial for each $i$ and consequently $\pi_{2}\left(\bigvee_{i=1}^{n} T_{i}\right)$ is trivial. Hence, for exactly one simple closed curve in the boundary of $C$, the disk side of this simple closed curve contains $C$. Let $D_{C}$ be this disk in $S_{z}$ and define $f_{C}: S_{z} \rightarrow \bigvee_{i=1}^{n}\left(T_{i}, x_{i}\right)$ by $f_{C}(x)=o$ for $x \in D_{C}$ and $f_{C}(x)=f_{z}(x)$ for $x \notin D_{C}$. Then $f_{C}$ is homotopic to $f_{z}$ relative to $S_{z} \backslash D_{C}$. The simple closed curves of boundaries of $D_{C_{0}}$ and $D_{C_{1}}$ are disjoint for distinct components $C_{0}$ and $C_{1}$ of $f_{z}^{-1}(\{o\})$. Therefore, if $D_{C_{0}}$ and $D_{C_{1}}$ intersect, then $D_{C_{0}} \subseteq D_{C_{1}}, D_{C_{1}} \subseteq D_{C_{0}}$ or $D_{C_{0}} \cup D_{C_{1}}=S_{z}$. The last case implies that $S_{z}$ is a 2 -sphere, which contradicts $g\left(S_{z}\right) \geq 1$. Hence we have connected components $C_{j}(1 \leq j \leq m)$ of $f^{-1}(\{o\})$ such that $D_{C_{j}} \cap D_{C_{j^{\prime}}}=\emptyset$ for $j \neq j^{\prime}$ and for each connected component $E$ of $f_{z}^{-1}(\{o\})$ there exists $C_{j}$ such that $D_{E} \subseteq D_{C_{j}}$. Now define $\bar{f}: S_{z} \rightarrow \bigvee_{i=1}^{n}\left(T_{i}, x_{i}\right)$ by $f(x)=o$ for $x \in \bigcup_{j=1}^{m} D_{C_{j}}$ and $\bar{f}(x)=f_{z}(x)$ otherwise. Then $\bar{f}$ is homotopic to $f_{z}$. Since $D_{C_{j}}$ are pairwise disjoint disks, $S_{z} \backslash \bigcup_{j=1}^{m} D_{C_{j}}$ is connected, which implies that the range of $\bar{f}$ is contained in some $T_{i}$. This contradicts the fact that $\left(r_{i} \circ f_{z}\right)_{*}$ is essential for $1 \leq i \leq k+1$ with $k \geq 1$. We have shown that there exists an essential closed curve $L$ in $f_{z}^{-1}(\{o\}$ ) (we may suppose that this curve is piecewise linear).

We cut open $S_{z}$ along $L$ and paste two disks. We have a cycle $z^{\prime}$ such that $z^{\prime}$ is homologous to $z$ and $f_{z^{\prime}}$ extends $f_{z}$ so that $f_{z^{\prime}}$ takes the value $o$ on these disks.

CASE 1: $S_{z^{\prime}}$ is connected.

Since $g\left(S_{z^{\prime}}\right)=g\left(S_{z}\right)-1$, the cardinality of $\left\{i: r_{i *}\left(z^{\prime}\right) \neq 0\right\}$ is at most $g\left(S_{z}\right)-1$. Since $z$ is homologous to $z^{\prime}$, we have a contradiction.

CASE 2: $S_{z^{\prime}}$ has two connected components.

We have two cycles $z_{0}$ and $z_{1}$ such that $z_{0}+z_{1}$ is homologous to $z^{\prime}, S_{z_{0}}$ and $S_{z_{1}}$ are connected closed surfaces. Then we have $g\left(S_{z_{0}}\right)+g\left(S_{z_{1}}\right)=g\left(S_{z}\right)$. Since $L$ is essential, $g\left(S_{z_{0}}\right), g\left(S_{z_{1}}\right) \geq 1$ and consequently $g\left(S_{z_{0}}\right), g\left(S_{z_{1}}\right)<$ $g\left(S_{z}\right)$. Now there exists $i_{0}$ such that the both $r_{i_{0} *}\left(\left[z_{0}\right]\right)=0$ and $r_{i_{0} *}\left(\left[z_{1}\right]\right)=0$, which contradicts $r_{i_{0} *}\left(\left[z_{0}\right]\right)+r_{i_{0} *}\left(\left[z_{1}\right]\right)=r_{i_{0} *}([z]) \neq 0$.

COROLlary 5.2. Let $z$ be a singular 2 -cycle of $\widetilde{\bigvee}_{i \in \mathbb{N}}\left(T_{i}, x_{i}\right)$. Then the cardinality of $\left\{i \mid r_{i *}([z]) \neq 0\right\}$ is at most $g\left(S_{z}\right)$.

Proof. To show this by contradiction, suppose that the cardinality of $\left\{i \mid r_{i *}([z]) \neq 0\right\}$ is greater than $g\left(S_{z}\right)$. We have $F \subseteq \mathbb{N}$ such that the cardinality of $F$ is greater than $g\left(S_{z}\right)$ and $r_{i *}([z]) \neq 0$ for every $i \in F$. Let $r_{F}: \widetilde{\bigvee}_{i \in \mathbb{N}}\left(T_{i}, x_{i}\right) \rightarrow \bigvee_{i \in F}\left(T_{i}, x_{i}\right)$ be the retraction projecting every torus $T_{j}$ with $j \notin F$ to $o$. We remark that we can construct $S_{r_{F \#}(z)}$ for $r_{F \#}(z)$ 
with $S_{r_{F \#}(z)}=S_{z}$. Since $r_{i *}\left(\left[r_{F \#}(z)\right]\right)=r_{i *}([z])$ for $i \in F$, the cardinality $\left\{i \in F: r_{i *}\left(\left[r_{F \#}(z)\right]\right) \neq 0\right\}$ is greater than $g\left(S_{r_{F \#}(z)}\right)$ which contradicts Lemma 5.1.

Proof of Theorem 1.4. First we recall the following Mayer-Vietoris sequence for $S C(T)$ :

$$
H_{2}\left(p_{2}^{-1}((0,1))\right) \stackrel{i_{0 *}-i_{1 *}}{\longrightarrow} H_{2}\left(p_{2}^{-1}([0,1))\right) \oplus H_{2}\left(p_{2}^{-1}((0,1])\right) \stackrel{j_{0 *}+j_{1 *}}{\longrightarrow} H_{2}(S C(T)),
$$

where $i_{0}: p_{2}^{-1}((0,1)) \rightarrow p_{2}^{-1}([0,1)), i_{1}: p_{2}^{-1}((0,1)) \rightarrow p_{2}^{-1}((0,1]), j_{0}:$ $p_{2}^{-1}([0,1)) \rightarrow S C(T)$ and $j_{1}: p_{2}^{-1}((0,1]) \rightarrow S C(T)$ are the inclusions. We have deformation retractions $r_{0}: p_{2}^{-1}([0,1)) \rightarrow M_{0}, r_{1}: p_{2}^{-1}((0,1]) \rightarrow M_{1}$ and $r_{1 / 2}: p_{2}^{-1}((0,1)) \rightarrow M_{1 / 2}$. We observe that $M_{0}, M_{1}$ and $M_{1 / 2}$ are homotopy equivalent to the Hawaiian tori wedge, more precisely, by the local contractiblity of tori we have the homotopy equivalences

$$
\begin{aligned}
M_{0} & \simeq \widetilde{V}_{n \in \mathbb{N}} p^{-1}\left(\left\{A_{n}\right\}\right), \\
M_{1} & \simeq \widetilde{V}_{n \in \mathbb{N}} p^{-1}\left(\left\{B_{n}\right\}\right), \\
M_{1 / 2} & \simeq \widetilde{V}_{n \in \mathbb{N}} p^{-1}\left(\left\{C_{n}\right\}\right) .
\end{aligned}
$$

Let $c_{A_{k}}: M_{0} \rightarrow p^{-1}\left(\left\{A_{k}\right\}\right)$ be the retraction so that $c_{A_{k}}\left(M_{0} \backslash p^{-1}\left(\left\{A_{k}\right\}\right)\right)=$ $\left\{A_{k}\right\}$ and let $c_{B_{k}}: M_{1} \rightarrow p^{-1}\left(\left\{B_{k}\right\}\right)$ and $c_{C_{k}}: M_{1 / 2} \rightarrow p^{-1}\left(\left\{C_{k}\right\}\right)$ be similar retractions (see Figure 1). Finally, let $r_{A_{k}}=c_{A_{k}} \circ r_{0}, r_{B_{k}}=c_{B_{k}} \circ r_{1}$ and $r_{C_{k}}=c_{C_{k}} \circ r_{1 / 2}$.

Since $p^{-1}\left(\left\{C_{1}\right\}\right)$ is homeomorphic to $T$, we let $z$ be the standard cycle such that $[z]$ is the generator of $H_{2}\left(p^{-1}\left(\left\{C_{1}\right\}\right)\right.$. We'll show that $[z]$ is nontrivial as an element of $H_{2}(S C(T))$. To show this by contradiction we suppose that $[z]=0$ in $H_{2}(S C(T))$. Since $z$ is a cycle in $p_{2}^{-1}([0,1))$, we have $[z]_{0} \in$ $H_{2}\left(p_{2}^{-1}([0,1))\right.$, where $[*]_{0}$ denotes a homology class in $H_{2}\left(p_{2}^{-1}([0,1))\right.$. Since $j_{0 *}\left([z]_{0}\right)+j_{1 *}(0)=[z]=0$, we have $u \in H_{2}\left(p_{2}^{-1}((0,1))\right)$ such that $i_{0 *}(u)=[z]_{0}$ and $-i_{1 *}(u)=0$.

Then we have $r_{A_{1} *} \circ i_{0 *}(u)=r_{A_{1} *}\left([z]_{0}\right)=1$ and $r_{A_{k} *} \circ i_{0 *}(u)=$ $r_{A_{k} *}\left([z]_{0}\right)=0$ for $k \geq 2$ and $r_{B_{k^{*}}} \circ i_{1 *}(u)=-r_{B_{k} *}(0)=0$ for $k \in$ $\mathbb{N}$. Consider the above homotopy equivalences of $M_{0}$ and $M_{1 / 2}$ together with the deformation retractions $r_{0}$ and $r_{1 / 2}$. Then we can see that $H_{2}\left(p^{-1}\left(\left\{C_{2 k-2}\right\}\right)\right) \oplus H_{2}\left(p^{-1}\left(\left\{C_{2 k-1}\right\}\right)\right)$ is a summand of $H_{2}\left(p^{-1}((0,1))\right)$ and $H_{2}\left(p^{-1}\left(\left\{A_{k}\right\}\right)\right)$ is a summand of $H_{2}\left(p^{-1}([0,1))\right)$. Therefore we can see that $i_{0 *} \mid H_{2}\left(p^{-1}\left(\left\{C_{2 k-2}\right\}\right)\right)$ and $i_{0 *} \mid H_{2}\left(p^{-1}\left(\left\{C_{2 k-1}\right\}\right)\right)$ are isomorphisms onto $H_{2}\left(p^{-1}\left(\left\{A_{k}\right\}\right)\right)$ for $k \geq 2$ and similarly $i_{1 *} \mid H_{2}\left(p^{-1}\left(\left\{C_{2 k-1}\right\}\right)\right)$ and $i_{1 *} \mid H_{2}\left(p^{-1}\left(\left\{C_{2 k}\right\}\right)\right)$ are isomorphisms onto $H_{2}\left(p^{-1}\left(\left\{B_{k}\right\}\right)\right)$ for $k \in \mathbb{N}$ and hence we have $r_{C_{1} *}(u)=1$ and $r_{C_{2} *}(u)=-1$ and successively $r_{C_{2 k+1} *}(u)=1$ and $r_{C_{2 k+2} *}(u)=-1$ for $k \in \mathbb{N}$. Since $u \in H_{2}\left(p_{2}^{-1}((0,1))\right)$, we have 
a singular 2-cycle $z_{0}$ of $p_{2}^{-1}((0,1))$ with $\left[z_{0}\right]=u$, where $p_{2}^{-1}((0,1))$ is homotopy equivalent to $\widetilde{\bigvee}_{i \in \mathbb{N}}\left(T_{i}, x_{i}\right)$. According to the description preceding Lemma 5.1 we have a closed surface $S_{z_{0}}$. By Corollary 5.2 the cardinality of $\left\{i \mid r_{C_{i} *}((u)) \neq 0\right\}$ is at most $g\left(S_{z_{0}}\right)$, which is a contradiction.

Next we consider the following property of a space:

There exists a point $x$ such that any neighborhood of $x$ contains an image of a nontrivial 2-cycle.

This property is homotopy invariant.

Since $p_{2}^{-1}([0,1))$ and $p_{2}^{-1}((0,1])$ are contractible by Lemma 4.1, $C A C(T)$ does not have this property. But for $C S C(T)$ any neighborhood of a point $(0 ; y)$ contains an image of a 2-cycle which is homologous to the standard 2cycle $z$, which is nontrivial. We have thus shown that $C S C(T)$ and $C A C(T)$ are not homotopy equivalent.

REMARK 5.3. For the proof of Theorem 1.4 a weaker assertion than Lemma 5.1 is sufficient. What is actually necessary is a bound on the cardinality of $\left\{i \mid r_{i *}([z]) \neq 0\right\}$ and this bound can be obtained by a surgery, i.e., by cutting open some $f^{-1}\left(T_{i}\right)$ instead of $f^{-1}(\{o\})$ in the proof of Lemma 5.1, which is easier. Since Lemma 5.1 itself is a basic fact, we have stated this exact form.

\section{Proof of Theorem 1.6}

Proof of Theorem 1.6. The statement (1) of Theorem 1.6 is a corollary of [2, Theorem A.1] and so we explain the notions and results around it. The free $\sigma$-product of groups, which is a subgroup of the inverse limit of finite free products of groups $([10])$, is defined by using countably infinite words as a generalization of the usual free products ([2, Definition 1.2]). We refer the reader to $[2$, Definition 1.2$]$ for a precise definition. Then for locally strongly contractible spaces $X_{n}, \pi_{1}\left(\widetilde{\mathrm{V}}_{n \in \mathbb{N}}\left(X_{n}, x_{n}\right)\right)$ is isomorphic to the free $\sigma$-product of $\pi_{1}\left(X_{n}, x_{n}\right)$ by [2, Theorem A.1]. Thus we have shown (1).

Next we show (2). Let $o$ be the distinguished point of the Hawaiian tori wedge $\mathbb{H}_{T}$ and $T_{n}$ be the $n$-th factor of $\mathbb{H}_{T}$. Let $f: \mathbb{I}^{2} \rightarrow \mathbb{H}_{T}$ be a continuous map such that $f\left(\partial \mathbb{I}^{2}\right)=\{o\}$. We have the countable family $\mathcal{U}$ consisting of pairwise disjoint connected open sets such that $\mathbb{I}^{2} \backslash f^{-1}(\{o\})=\bigcup \mathcal{U}$. For each $U \in \mathcal{U}, f(U)$ is contained in some $T_{n}$. Since each torus $T_{n}$ is locally strongly contractible at $o$ and a neighborhood of $o$ contains almost all $T_{n}$, we may assume that $\left\{U \in \mathcal{U}: f(U) \subseteq T_{n}\right\}$ is finite for each $T_{n}$ and also that each $U \in \mathcal{U}$ is an open polygon. We remark that $U$ may have holes and it divides $\mathbb{I}^{2}$ into finitely many, possibly only one, connected components. For each $U \in \mathcal{U}$, let $D_{U}$ be the open disk such that $\mathbb{I}^{2} \backslash D_{U}$ is the unique connected component of $\mathbb{I}^{2} \backslash U$ containing $\partial \mathbb{I}^{2}$. Then we have $U \subseteq D_{U}$. For $U, V \in \mathcal{U}$, we define $U \prec V$, if $U$ is contained in $D_{V}$ but $V$ is not contained in $D_{U}$. 
Since $\prec$ is a partial order and $\mathcal{U}$ is at most countable, we have an orderpreserving map $\rho: \mathcal{U} \rightarrow \mathbb{Q}$. The complement of the Cantor ternary set consists of a disjoint union of open intervals and the disjoint intervals are ordered naturally and this ordering is isomorphic to that of the rationals $\mathbb{Q}$. We number the open intervals as $\left(a_{q}, b_{q}\right)$ by $q \in \mathbb{Q}$ so that $p<q$ implies $b_{p}<a_{q}$. For $U \in \mathcal{U}$ we define $f_{U}: \overline{D_{U}} \rightarrow \mathbb{H}_{T}$ such that $f_{U}(x)=f(x)$ for $x \in U$ and $f_{U}(x)=o$ for $x \in \overline{D_{U}} \backslash U$.

We define a homotopy $H: \mathbb{I}^{2} \times \mathbb{I}$ so that $H(x, 0)=f(x)$ for $x \in \mathbb{I}^{2}$, $H(x, t)=o$ for $x \in \partial \mathbb{I}^{2}, t \in \mathbb{I}$ and $H(x, 1)=o$ for $x \in \mathbb{I}^{2}$. For this purpose we define $H$ first on some parts.

For each $U \in \mathcal{U}$, we define $H(x, t)=f(x)$ for $x \in U$ and $t \leq a_{\rho(U)}$ and let $H \mid \overline{D_{U}} \times\left[a_{\rho(U)}, b_{\rho(U)}\right]$ to be a homotopy from $f_{U}$ to the constant map to $\{o\}$. Now, $H$ is continuous on $\overline{D_{U}} \times\left[a_{\rho(U)}, b_{\rho(U)}\right] \cup \bar{U} \times\left[0, a_{\rho(U)}\right]$ for each $U \in \mathcal{U}$.

According to the definitions of $\rho$ and $D_{U}$ the defined parts are overlapping only where the value of $H$ is $o$. To see this, suppose that $D(U) \cap D(V) \neq \emptyset$ for distinct $U$ and $V$. Then we have $D(U) \cap V \neq \emptyset$ or $D(V) \cap U \neq \emptyset$. Since $U$ and $V$ are disjoint and each of them is connected, we have $V \subseteq D(U)$ or $U \subseteq D(V)$ and hence $V \prec U$ or $U \prec V$. We only deal with the case $V \prec U$. Since $U \cap V=\emptyset$ and $b_{\rho(V)}<a_{\rho(U)}$, we have

$$
\left(D_{U} \times\left[a_{\rho(U)}, b_{\rho(U)}\right] \cup U \times\left[0, a_{\rho(U)}\right]\right) \cap\left(D_{V} \times\left[a_{\rho(V)}, b_{\rho(V)}\right] \cup V \times\left[0, a_{\rho(V)}\right]\right)=\emptyset
$$

and we conclude that the defined parts are overlapping only where the value of $H$ is $o$.

We define $H(x, t)=o$ for the remaining undefined parts. We need to show the continuity of $H$. A crucial matter is the accumulation of $H^{-1}\left(T_{n}\right)$ for infinitely many $n$, but we define the value of $H$ to be $o$ for such a point. Since each neighborhood of $o$ contains almost all $T_{n}$ and $\left\{U \in \mathcal{U}: f(U) \subseteq T_{n}\right\}$ is finite for each $T_{n}$, the continuity of $H$ is now clear. Since $H(x, 1)$ is defined as the remaining undefined parts, $H$ is a homotopy to the constant map to $\{o\}$, which implies that $f$ is null-homotopic.

In the remaining part we shall prove (3). Let $f$ be a continuous map from a closed surface $S_{z}$ to $\mathbb{H}_{T}$. Let $r_{i}$ be the retraction of $\mathbb{H}_{T}$ to the $i$-th factor. By Corollary 5.2 $r_{i} \circ f$ is null-homologous for almost all $i$. As in the proof of (2) we may suppose that each connected component of $f^{-1}\left(\mathbb{H}_{T} \backslash\{o\}\right)$ is an open subsurface. Next we find a simple closed curve in $f^{-1}(\{o\})$ which is essential in the surface. We cut open along the simple closed curve. We now iterate this procedure.

Hence we have $g_{0}, \cdots, g_{n}$ such that

(a) the domain $S_{z_{i}}$ of each $g_{i}$ is a connected closed surface;

(b) the singular cycle $z_{1}+\cdots+z_{n}$ is homologous to $z$;

(c) every simple closed curve in $g_{i}^{-1}(\{o\})$ is null-homotopic in the surface; and 
(d) each connected component of $g_{i}^{-1}\left(\mathbb{H}_{T} \backslash\{o\}\right)$ is an open subsurface.

We fix $g_{i}$. Let $U$ be a connected component of $g_{i}^{-1}\left(\mathbb{H}_{T} \backslash\{o\}\right)$. Then by the property (c) every simple closed curve in the boundary of $U$ bounds a disk. Since the genus of $S_{z}$ is positive, at most one of those closed disks contains $U$. If there is no such disk, the complement of $U$ of the surface is covered by those finitely many disks. Then by Theorem 1.6(2) the restriction of $g_{i}$ to each disk is homotopic to the constant $o$ and hence we conclude that $g_{i}$ is homotopic to a map into a single $T_{j}$ for $j \in \mathbb{N}$. Otherwise, for each $U \in \mathcal{U}$ there exists an open disk $D_{U}$ which contains $U$ and whose boundary is a connected component of the boundary of $U$. Now we define $U \prec V$ if $U \subseteq D_{V}$, but $V \nsubseteq D_{U}$ in a similar way as in the proof of (2).

If $D_{U}$ contains $V$ and $D_{V}$ contains $U$, then we have $U=V$. To see this by contradiction, suppose that $U \neq V$. Then, since $U \cap V=\emptyset$ and $\partial D_{V}$ is a connected component of $\partial V$, we have $S_{z_{i}} \backslash D_{U} \subseteq D_{V}$ and this implies that $S_{z_{i}}$ is a sphere, which is a contradiction. Hence $U \prec V$ is a partial order as in the proof of (2). In addition, since $S_{z_{i}}$ is a surface, for distinct $U$ and $V$, $D(U) \cap D(V) \neq \emptyset$ implies $D(U) \cap V \neq \emptyset$ or $D(V) \cap U \neq \emptyset$ as in (2) and consequently $V \prec U$ or $U \prec V$. Now we continue as in the proof of (2). The difference is that the domain is a closed surface $S_{z_{i}}$ instead of a square, but the proof is formally the same and we have a homotopy from $g_{i}$ to the constant map to $\{o\}$. Now all of the above implies that $[z] \in \oplus_{i \in \mathbb{N}} H_{2}\left(T_{i}\right) \cong \oplus_{\mathbb{N}} \mathbb{Z}$. Since $\oplus_{i \in \mathbb{N}} H_{2}\left(T_{i}\right) \leq H_{2}\left(\mathbb{H}_{T}\right)$, we have

$$
H_{2}\left(\mathbb{H}_{T}\right)=\oplus_{i \in \mathbb{N}} H_{2}\left(T_{i}\right) \cong \oplus_{\mathbb{N}} \mathbb{Z}
$$

We complete this paper with the following three interesting problems which remain open:

Problem 6.1. Is the group $H_{3}\left(\mathbb{H}_{T}\right)$ trivial?

Problem 6.2. Does there exist a 2-dimensional Peano continuum which is cell-like, simply connected, noncontractible, and aspherical in dimension 2?

Problem 6.3. Does there exist a finite-dimensional noncontractible Peano continuum whose homotopy groups are trivial?

ACKNOWLEDGEMENTS.

This research was supported by the Slovenian Research Agency grants P10292-0101-04, J1-4144-0101-07, J1-2057-0101 and the Polish-Slovenian grant BI-Pl-2010-2011-001. The first author was partially supported by the Grantin-Aid for Scientific research (C) of Japan No. 20540097 and 23540110.

\section{REFERENCES}

[1] G. Conner, M. Meilstrup, D. Repovš, A. Zastrow and M. Željko, On small homotopies of loops, Topology Appl. 155 (2008), 1089-1097. 
[2] K. Eda, Free $\sigma$-products and non-commutatively slender groups, J. Algebra 148 (1992), 243-263

[3] K. Eda, U. H. Karimov and D. Repovš, A construction of noncontractible simply connected cell-like two-dimensional Peano continua, Fundamenta Math. 195 (2007), 193-203.

[4] K. Eda, U. H. Karimov and D. Repovš, A nonaspherical cell-like 2-dimensional simply connected continuum and related constructions, Topology Appl. 156 (2009), 515-521.

[5] K. Eda, U. Karimov and D. Repovš, The second homotopy group of $S C(Z)$, Glas. Mat. Ser. III 44(64) (2009), 493-498.

[6] K. Eda, U. Karimov and D. Repovš, On the singular homology of one class of simplyconnected cell-like spaces, Mediterr. J. Math. 8 (2011), 153-160.

[7] K. Eda, U. H. Karimov and D. Repovš, On 2-dimensional nonaspherical cell-like Peano continua. A simple approach, Mediterranean J. Math. 10 (2013), 519-528.

[8] K. Eda and K. Kawamura, Homotopy and homology groups of the $n$-dimensional Hawaiian earring, Fund. Math. 165 (2000), 17-28.

[9] A. Hatcher, Algebraic Topology, Cambridge Univ. Press, Cambridge, 2002.

10] G. Higman, Unrestricted free products and varieties of topological groups, J. London Math. Soc. 27 (1952), 73-81.

[11] E. H. Spanier, Algebraic Topology, McGraw-Hill, New York-Toronto, 1966.

K. Eda

School of Science and Engineering

Waseda University

Tokyo 169-8555

Japan

E-mail: eda@logic.info.waseda.ac.jp

\section{U.H. Karimov}

Institute of Mathematics

Academy of Sciences of Tajikistan

Ul. Ainy $299^{A}$, Dushanbe 734063

Tajikistan

E-mail: umedkarimov@gmail.com

D. Repovš

Faculty of Education, and Faculty of Mathematics and Physics

University of Ljubljana

P.O.Box 2964, Ljubljana 1001

Slovenia

E-mail: dusan.repovs@guest.arnes.si

\section{A. Zastrow}

Institute of Mathematics

Gdansk University

ul. Wita Stwosza 57, 80-952 Gdańsk

Poland

E-mail: zastrow@mat.ug.edu.pl

Received: 29.10.2011.

Revised: 17.8.2012. 